\title{
Section: Review
}

\section{Oxidative stress in fungal fermentation processes: the roles of alternative respiration}

Li Q ${ }^{1}$, Bai $Z^{2}$, O’Donnell $\mathrm{A}^{3}$, Harvey $\mathrm{LM}^{4}$, Hoskisson, $\mathrm{PA}^{4}$ and $\mathrm{McNeil} \mathrm{B}\left({ }^{*}\right)^{4}$

${ }^{1}$ Dept Biochemical Engineering, University College London, ${ }^{2}$ GSK, Stevenage, Herts ; ${ }^{3}$ Millipore Ltd, Livingstone, Midlothian ; ${ }^{4}$ Strathclyde Institute for Biomedical Science, University of Strathclyde, Glasgow (*) Corresponding author 01415522562 b.moneil@strath.ac.uk

Key words: alternative NADH dehydrogenases; alternative oxidase; alternative respiratory pathway; fungi; metabolism; oxidative stress 


\begin{abstract}
Filamentous fungi are arguably the most industrially important group of microorganisms. Production processes involving these simple eukaryotes are often highly aerobic in nature, which implies these cultures are routinely subject to oxidative stress. Despite this, little is known about how filamentous fungi cope with high levels of oxidative stress as experienced in fermenter systems. More surprisingly, much of our knowledge of oxidative stress responses in fungi comes from environmental or medical studies. Here, the current understanding of oxidative stress effects and cellular responses in filamentous fungi is critically discussed. In particular the role of alternative respiration is evaluated, and the contributions of the alternative oxidase and alternative dehydrogenases in defence against oxidative stress, and their profound influence on fungal metabolism is critically examined. Finally, the importance of further research which would underpin a less empirical approach to optimising fungal strains for the fermenter environment is emphasised.
\end{abstract}

\title{
Introduction
}

Fungi, which consist of both the yeasts and the filamentous fungi, are among the most widely used microorganisms in traditional and advanced bio-industries. In the past decade, novel yeast strains and filamentous fungi have been progressively invading the field of bio-pharmaceutical 
protein expression dominated by Escherichia coli and mammalian cell expression systems (Sodoyer 2004).

In terms of synthesis of heterologous proteins, filamentous fungi have a complex posttranslational protein modification system, unlike the simpler bacterial systems which tend to be used for production of non-glycosylated proteins (Schmidt 2004). The fungal species preferred for recombinant protein expression are those widely employed in traditional processes for manufacturing enzymes, acids and antibiotics, and these include species from a few key genera including Aspergillus, Penicillium, Acremonium, Fusarium and Trichoderma. Since filamentous fungi typically obtain the nutrients they require by secretion of digestive enzymes and subsequent absorption of the digested materials, the innate protein productivity and secretion potential of these filamentous fungal hosts are often far superior to other expression systems. However, even some yeasts, in particular Saccharomyces cerevisiae and Pichia pastoris, have been used successfully to express a multitude of recombinant proteins, such as Fab (fragment antibody) and ScFv (single chain variable fragment) therapeutic antibodies (Schoonooghe et al. 2009; Yamawaki et al. 2007) and protein vaccines (Avril et al. 2009), since they posses many of the bioprocessing advantages of E. coli. For example, short doubling times and readily manipulated genomes, but also have the additional benefits of eukaryotes such as improved protein folding and many post- translational modifications (Durocher \& Butler 2009; Yurimoto \& Sakai 2009). Furthermore, filamentous fungi and yeasts are more robust than mammalian cells and can produce some enzymes in excess of $100 \mathrm{~g} / \mathrm{l}$ in fermenters (Cherry 2003) and are easily adapted to fermentation processes in simple and cost-effective culture media (Gasser and Mattanovich 2007; Durocher \& Butler 2009). Even though filamentous fungi and yeasts have now been used industrially for decades, and are now used for production of some of our most 
complex and potent drugs, they are still not adequately characterized at the physiological and genetic level, particularly in terms of protein modification and secretion metabolism (Durocher \& Butler 2009) and especially in relation to their physiological responses to the stressful growing environment in bioreactors (Li et al. 2009 ; Bai et al. 2003a).

The term "stress" is widely used, and is somewhat controversial, but it is also very difficult to define in objective terms. "Oxidative stress" is a very frequently encountered term, so at the outset this term should be clearly defined. Oxidative stress can be simply defined as a state where there is a relative imbalance within cells between generation of reactive oxygen species (ROS) and the intracellular anti-oxidant defences in favour of the former ( Li et al. 2009). Most fungal processes are highly aerobic in nature, and thus sufficient $\mathrm{O}_{2}$ supply and the maintenance of $\mathrm{O}_{2}$ transfer rate in the bioreactor is always a challenge during production processes, and especially in those processes involving high cell density. As a solution, the use of $\mathrm{O}_{2}$-enriched air is frequently applied in many industrial fungal processes (Bai et al. 2003a). However, $\mathrm{O}_{2}$ can be a two-sided sword for microbial cells inside bioreactors, mainly due to the generation of ROS, which is of particular relevance to the cultivation of industrial fungi. A clear understanding of how fungal cells cope with the challenges of toxic ROS is, thus, critical for improved insight into fungal physiology. To a large extent, this is consistent with the regulatory view that we should seek to better understand our bio-manufacturing processes. Unfortunately our knowledge on many aspects of oxidative stress in fungal processes is still deficient in many areas. To fully exploit the potential of filamentous fungi and yeast, their physiology and particularly the mechanisms of defensive responses to such a highly aerobic growing environment has to be investigated and clarified. Recent advances in this important field are discussed here, with an emphasis on the implications of the findings of these studies to fungal bio-processing. 
Oxidative stress is the unavoidable consequence of an aerobic lifestyle. We, as biotechnologists, operate fungal bioproduction processes with very high $\mathrm{O}_{2}$ transfer rates, and occasionally $\mathrm{O}_{2}$ enrichment in order to achieve high productivities. Thus, as an industry we take for granted a set of conditions which impose, at some process time points, intense oxidative stress on the fungal production and expression systems we employ. Despite this, it is astounding that we have spent little time or effort trying to understand clearly how the fungi we use in bioreactors deal with the conditions we impose. This leaves us in the realm of empiricism in process definition and operation. Much of what knowledge we possess about fungal defence against oxidative stress comes not from fermenter studies, but from environmental and medical mycology, and often uses relatively convenient chemical stressors, e.g. simple addition of exogenous hydrogen peroxide to cultures, to simulate actual oxygen induced stress (as in fermenters), thus, the relevance to aerobic fermentations of fungi is questionable. Our first need is, therefore, for more carefully though- out studies focused upon fungal bioproduction systems and the process consequences of oxidative stress.

\section{General responses to oxidative stress in fungi}

There is some evidence suggesting that oxidative stress imposes significant effects on fungal cultures in bioproduction systems, in terms of morphology, growth rate, metabolism, and protein secretion. This review will briefly summarise the findings of such studies and then discuss an increasingly important but generally neglected area, i.e. the role of alternative respiratory 
enzymes in oxidative stress. For more comprehensive discussions, readers are encouraged to refer to a recent review by our group (Li et al. 2009).

The effects of oxidative stress on fungal morphology

Filamentous fungi exhibit two extreme types of morphology in submerged culture, pelleted and filamentous forms. Gassing batch cultures of A. niger with $\mathrm{O}_{2}$-enriched air decreases the size of hyphal elements and increases the proportion of active cytoplasm, but hyphal adhesion occurs at the highest level of $\mathrm{O}_{2}$ enrichment (50\% v/v) (Wongwicharn et al. 1999). Similar results have been found where $\mathrm{O}_{2}$ enrichment results in branched and shorter hyphae (Kreiner et al. 2003; Bai et al. 2004). Addition of exogenous $\mathrm{H}_{2} \mathrm{O}_{2}$ leads to a decrease of the hyphal growth unit (Kreiner et al. 2003). In yeast, a study showed morphological changes from unicellular to pseudomycelium in the yeast Ustilago maydis when treated with a pro-oxidant compound, octyl gallate (Sierra-Campos et al. 2009). Substantial evidence therefore exists to indicate that morphological change may be a direct consequence of oxidatively stressful conditions in the fungi.

The effects of oxidative stress on fungal growth rates and metabolism

Oxidative stress can be provoked by addition of exogenous chemical stressors (simulated) or by highly aerobic conditions within a fermenter system, for example, by oxygen enrichment of the gas phase. Oxidative stress generally exerts a negative impact on fungal growth. This was shown by the prolonged lag phase noted in cultures transferred from shake flasks to a highly aerated and well mixed fermenter system (O'Donnell et al. 2007), slow growth rates, low substrate 
consumption rates (O'Donnell et al. 2007; Li et al. 2008a), low protein content (Bai et al. 2004) and low ATP content ( $\mathrm{Li}$ et al. 2008b) in oxidatively-stressed fungal cells. One possible explanation for many of these observations is the engagement of alternative respiration, one of whose putative roles is to diminish ROS production, but at the expense of ATP generation due to their non-proton-pumping nature. Another possible explanation is the specific inhibition of key metabolic enzymes by ROS. Two frequently studied enzymes that are vulnerable to ROS inactivation are glyceraldehyde-3-phosphate dehydrogenase (Li et al. 2008a) and aconitase (Murakami and Yoshino 1997). Both these enzymes are essential for primary metabolism in fungi. Our results indicate that the decreased glucose uptake rate in A. niger correlates closely with deceased glyceraldehyde-3-phosphate dehydrogenase activity when cells are treated with $\mathrm{H}_{2} \mathrm{O}_{2}$, but that the decreased ammonium uptake rate is due to decreased ATP and NAPDH availability rather than oxidative inactivation of the ammonium assimilatory enzymes in cells (Li et al. 2008a).

In order to alleviate oxidative stress that is caused to the cells during bioprocessing, there have been some attempts to generate fungal mutants with increased antioxidant capacity. Such studies have a measure of success, for example, in over-expression of genes coding for antioxidant enzymes such as catalase (Abbott et al. 2009) and thioredoxin (Perez-Torrado et al. 2009), introduction of genes for synthesis of antioxidants such as vitamin C (Branduardi et al. 2007) and astaxanthin (Ukibe et al. 2009) all increase yeast cells' resistance to oxidative stress. Provided the genetic alterations made to these strains do not reduce vigour, or lead to altered proteolysis, such mutants could have clear advantages in industrial fermentation where oxidative stress is encountered.

The effects of oxidative stress on enzyme production in fungi 
Oxidative stress, caused by over-oxygenation to bioreactors, adversely affects the production of a recombinant protein from A. niger (Wongwicharn et al. 1999; Bai et al. 2004). Thus, mitigating oxidative damage could ultimately increase protein production by fungi. Overexpression of the Kluyveromyces lactis SOD1 (KlSOD1) gene increased the production of two different heterologous proteins by $K$. lactis, human serum albumin (HSA) and Arxula adeninivorans glucoamylase (Raimondi et al. 2008). This was thought to be due to the alleviation of the oxidative stress caused by sustained endoplasmic reticulum damage during recombinant protein production. Sustained stress of the endoplasmic reticulum (ER) causes oxidative stress both in yeast and mammalian cells (Harding et al. 2003; Haynes et al. 2004)). Overexpression of SOD alleviated oxidative stress, thus promoting recombinant protein production.

Conversely, oxidative stress may contribute to increased enzyme production under certain circumstances, for example, enhanced extracellular laccase activity is found as a part of the response system of white rot fungi, Trametes versicolor and Abortiporus biennis, to paraquatcaused oxidative stress conditions (Jaszek et al. 2006).

Having briefly outlined the general responses of fungi to oxidative stress, the discussion will now centre upon the central role of the alternative respiratory pathways in oxidative stress.

\section{Alternative respiratory pathways}

In the fungi, as in other eukaryotes, the cytochrome respiratory chain consists of four protein complexes: Complex I (NADH:ubiquinone oxidoreductase), Complex II (succinate:ubiquinone 
oxidoreductase), Complex III (ubiquinone:cytochrome $c$ oxidoreductase), Complex IV (cytochrome c oxidase). During electron transfer, energy is conserved by pumping out protons at Complexes I, III, IV across the inner membrane of mitochondria. Each complex has specific inhibitors, such as rotenone and piericidin for complex I, antimycin for complex III and cyanide for complex IV (Joseph-Horne et al. 2000). Complex II does not pump protons due to the low free energy change during respiration.

The alternative respiratory and electron transfer chain

[Figure 1 here]

In addition to Complex I, most plants and many fungi have alternative NADH dehydrogenases which accept electrons from NADH and transfer them to ubiquinone directly ( Fig 1). However, these enzymes do not pump protons out of the matrix across the inner mitochondrial membrane, and the free energy between $\mathrm{NADH}$ and ubiquinone $(73 \mathrm{~kJ} / 2 \mathrm{e}-$ ) is lost as heat (Siedow and Umbach 1995). Unlike Complex I, alternative NADH dehydrogenases are insensitive to rotenone, a fact that is often used to estimate the alternative respiratory activity by inhibiting the cytochrome pathway using rotenone.

In addition to the alternative dehydrogenase, there is also an alternative oxidase (AOX) which transfers electrons from ubiquinol to the final acceptor, $\mathrm{O}_{2}$, bypassing both Complex III and IV (Fig.1). As with the alternative dehydrogenases, AOX does not translocate protons when electrons flow through this pathway, thus no energy is conserved but is instead again lost as heat (Moore et al. 2002). This enzyme has been found in the inner membrane of mitochondria from 
all the higher plants, many algae, fungi, yeast and certain protozoa (Moore et al. 2002 ; Baurain et al. 2003 ; Juarez et al. 2006). It is distinguished from the conventional cytochrome pathway by its insensitivity to cyanide, which inhibits cytochrome $c$ oxidase (complex IV), and sensitivity to salicylhydroxamic acid (SHAM) and n-propyl gallate. These characteristics are often used to measure the activity of AOX in intact cells or isolated mitochondria by measuring $\mathrm{O}_{2}$ uptake rate in the presence of cyanide and/or SHAM.

Regulation of AOX in fungi.

\section{Expression of AOX genes is constitutive}

In Magnaporthe grisea (Yukioka et al. 1998), Neurospora crassa (Tanton et al. 2003) and A. niger (Hattori et al. 2009), AOX genes were constitutively transcribed at a low basal level even though the protein and enzyme activity were not detectable. The observation that non-induced wild-type cultures occasionally contained similar amounts of aod-1 mRNA to induced cultures but revealed no detectable $\mathrm{AOX}$ in mitochondria of $N$. crassa suggested translational control is also indispensable for the regulation of gene expression (Tanton et al. 2003).

AOX is not stimulated by pyruvate but by purine nucleotides

In contrast to plants, the fungal $\mathrm{AOX}$ is not stimulated by pyruvate, but by purine nucleotide monophosphate and diphosphates like AMP, ADP, dAMP and GMP. Evidence for this has been found in a wide range of yeasts and fungi, such as P. stipitis, N. crassa (Umbach and Siedow 2000), U. ayclis (Juarez et al. 2004), U. maydis (Sierra-Campos et al. 2009), Tapesia acuformis 
(Joseph-Horne et al. 2000) and in the yeasts Hansenula anomala (Sakajo et al. 1997) and Yarrowia lipolytica (Medentsev et al. 2004).

\section{AOX is activated in the presence of cytochrome respiration inhibitors}

Numerous studies have shown that AOX is activated when antimycin (an inhibitor of Complex III in the main respiratory chain), is added to fungal cultures (Minagawa et al. 1992; Uribe and Khachatourians 2008; Sierra-Campos et al. 2009). This property indicates that AOX overflows electrons when the cytochrome respiratory chain is inhibited or saturated. In the pathogenic fungus, U. maydis, $\mathrm{AOX}$ is thought to increase the metabolic flexibility of the cell, a role that might be valuable for an organism exposed to variations in temperature, nutrient source and availability, and biotic or abiotic factors that limit the activity of the cytochrome pathway (Juarez et al. 2006).

\section{The role of $A O X$ in oxidative stress}

It is generally accepted that mitochondria are the site for the generation of ROS in fungi due to election leakage during the process of aerobic respiration ( $\mathrm{Li}$ et al. 2009). One of the putative roles of the fungal alternative respiratory pathways may be to diminish ROS generation due to the non-proton pumping nature of many of the components of this pathway. There is some evidence to support this hypothesis. In $M$. grisea, for example, active oxygen species are thought to be signal mediators to activate transcription of the AOX gene (Yukioka et al. 1998), and the superoxide anion $\left(\mathrm{O}_{2}{ }^{--}\right)$is involved in the induction of cyanide-resistant-respiration (CRR) in the yeast $H$. anomala (Minagawa et al. 1992). The presence of $\mathrm{H}_{2} \mathrm{O}_{2}$ and menadione 
induces CRR in Pichia membranifaciens and Debaryomyces hansenii (Veiga et al. 2003). Karrafa et al. (2001) reported a negative correlation between CRR and intracellular peroxide levels in $A$. chrysogenum, supporting the protective role of alternative respiration against oxidative damage in cells. The effects of two ROS-generating agents, paraquat and $\mathrm{H}_{2} \mathrm{O}_{2}$ were investigated in 12 fungal species, the results of which indicated exposure of fungal spores or mycelia to both agents stimulated CRR (Angelova et al. 2005). In A. fumigatus, AOX activity and mRNA expression were both induced with menadione or paraquat, suggesting an important role of AOX under oxidative stress conditions (Magnani et al. 2007). A recent study by our group showed that oxidative stress in batch cultures of A. niger caused by over-oxygenation led to a general decrease of ATP content in the cells ( $\mathrm{Li}$ et al. 2008b) which corroborates the engagement of alternative respiration under such conditions proposed by Bai et al. (2003b).

Though the role of AOX in oxidative stress has been extensively researched, most of the work is based on "simulated" oxidative stress events, that is using oxidants that are highly pro-oxidative to simulate an oxidative environment. Valuable as those studies undoubtedly are, the unambiguous role (or roles) of AOX in aerobic fungal fermentation processes is still elusive. To address this, a comparison between a wild type and a mutant that is deficient in AOX cultivated under fermentation conditions that are known to induce oxidative stress would be very helpful.

\section{Alternative NADH dehydrogenases}

In addition to the alternative oxidase (AOX), many filamentous fungi contain alternative $\mathrm{NADH}$ :ubiquinone oxidoreductase (NADH dehydrogenase) enzymes. These alternative NADH 
dehydrogenase enzymes do not contain characteristic Fe-S clusters and are insensitive to rotenone or amytal (Kerscher 2000). Likewise, the alternative NADH dehydrogenases do not contain FMN, instead incorporating flavin adenine dinucleotide (FAD) as their sole prosthetic group (Prömper 1993), and are, like AOX, unable to translocate protons (Videira \& Duarte 2002). As a result of the non-proton pumping nature of these alternative enzymes, fungi must avoid pairing alternative NADH dehydrogenases with the alternative oxidase, as this would result in complete dissolution of the proton gradient necessary for ATP synthesis (Joseph-Horne et al. 2001). Therefore, alternative NADH dehydrogenases must be used synergistically with Complex III and IV, whereas AOX must only be used alongside Complex I, if the cell is to maintain sufficient ATP synthesis to drive all cellular processes (Joseph-Horne et al. 2001). Therefore, no filamentous fungus has been shown to contain an electron transport chain in which AOX is present but Complex I is absent. In many filamentous fungi there are three alternative NADH dehydrogenases, two of which are oriented towards the intermembrane space and hence oxidise cytosolic NADH/NADPH ("external" enzymes), and another which faces the mitochondrial matrix and oxidises internal NADH ("internal" enzyme) (Prömper et al. 1993; Videira \& Duarte 2002).

Thus far, three alternative dehydrogenase enzymes have been characterised in $N$. crassa, two external and one internal. These are designated NDE1, NDE2, and NDI1, respectively. NDE1 is calcium-dependent and can oxidise NADPH in addition to NADH, as can NDE2, whereas NDI1 can oxidise internal NADH alone (Duarte et al. 2003; Carneiro et al. 2004). Of these, NDI1 is undoubtedly the most important protein complex, since inactivation of the ndil gene, which 
encodes the enzyme, has been shown to result in complete inhibition of respiratory activity in the presence of rotenone (Duarte et al. 2003).

There is marked contrast between the alternative NADH dehydrogenases of filamentous fungi and those of yeast, although some homology is conserved. The yeast Yarrowia lipolytica, for instance, contains only one alternative $\mathrm{NADH}$ dehydrogenase, in contrast with the numerous alternative respiratory enzymes of filamentous fungi such as $N$. crassa. This enzyme is designated NDH2, and is encoded by the YLNDH2 gene. It is oriented towards the external face of the mitochondrial inner membrane, and oxidises cytosolic NADH or NADPH (Kerscher et al. 1999). Conversely, S. cerevisiae contains no Complex I, instead relying on three alternative NADH:ubiquinone oxidoreductases, SCNDE1, SCNDE2, and SCNDI1, the first two of which are external and the latter internal (Kerscher 2000). No specific inhibitor of the alternative $\mathrm{NADH}$ dehydrogenases of fungi has yet been reported, although unspecific inhibitors include flavone, platanetin (6-dimethylallyl-3,5,7,8-tetrahydroxyflavone) (Kerscher 2000), and 7-iodoacridone 4-carboxylic acid (IACA) (Roberts et al. 1996). Polymyxin B and nanaomycin A also inhibit alternative NADH dehydrogenases in bacteria (Mogi et al. 2009), which provides further scope for investigation of these compounds against fungal NADH dehydrogenases with which the bacterial dehydrogenases share a degree of similarity (Duarte 2003).

Although at present the exact physiological role of alternative $\mathrm{NADH}$ dehydrogenases in filamentous fungi is obscure, numerous researchers have postulated that they may be involved in prevention of, or perhaps even contribute to, reactive oxygen species generation. As regards the prevention of oxidative stress, Complex I and Complex III are the major sites of ROS generation by the mitochondrial ETC, accounting for some $20 \%$ and $80 \%$ of total $\mathrm{O}_{2}{ }^{--}$production, respectively (Turrens et al. 1985; Sigler et al. 1999). Therefore, in cultures experiencing 
oxidatively stressful conditions, for example $\mathrm{O}_{2}$ enrichment, it would be expected that levels of ROS would rise due to an increased rate of respiration. However, Bai et al. (2003b) described a significantly decreased $\mathrm{O}_{2}{ }^{--}$generation in cultures of $A$. niger in $\mathrm{O}_{2}$-enriched air, and thencefore proposed that this may be due to enhanced activity of an alternative NADH dehydrogenase enzyme, which could act in place of Complex I. Upon studying the total and rotenone-insensitive respiration of these cultures, those sparged with $\mathrm{O}_{2}$-enriched air had markedly higher ratios of rotenone-insensitive:total respiration (RR/TR). The researchers therefore concluded that under oxidatively challenging conditions, filamentous fungi manipulate electron transport to favour flux through the alternative respiratory pathway, thus reducing ROS generation, a theory which has likewise been proposed for other organisms (Li \& Trush 1998; Guerrero-Castillo et al. 2009). Seo et al. (2006) demonstrated that transduction of human neuroblastoma cells with the gene encoding the internal alternative NADH dehydrogenase of $S$. cerevisiae significantly reduced the generation of superoxide radicals within the cell. Inhibition of fungal NDI1 with IACA also led to a significant increase in superoxide production by the culture, most likely as a result of the high rate of electron leakage from Complex I (O'Donnell 2007).

Despite the protective properties of alternative NADH dehydrogenases against oxidative stress, further investigation in A. niger has shown that when these enzymes are enhanced in their activities, intracellular ATP concentrations and cellular viability are decreased (O'Donnell et al. 2007). This was particularly true under conditions of high (50\%) oxygen enrichment, which led primarily to a significant decrease in intracellular ATP concentrations, with a concomitant decrease in both intracellular and secreted protein concentrations and decreased respiratory quotients (O'Donnell 2007). Additionally, inhibition of the alternative NADH dehydrogenases by 
IACA significantly increased the intracellular ATP concentrations, both in control and $\mathrm{O}_{2 \text { - }}$ enriched batch cultures (O'Donnell 2007). Other investigators have also claimed such alternative NADH dehydrogenase enzymes actually increase ROS production. Fang \& Beattie (2003) demonstrated that inhibition of alternative NADH dehydrogenases in yeast ( $S$. cerevisiae) led to a decrease in ROS production by the culture. The apparent contradiction could well be due to the differing responses of yeasts and filamentous fungi to oxidative stress. (Li 2009).

Since 2005, the genomes have been available for A. nidulans, A. fumigatus and A. oryzae (Jones 2007), with the genome for A. niger being determined by DSM (The Netherlands) and made publicly available in 2007 (Pel et al. 2007). The public availability of these genomes brings with it further opportunity to understand the functions of each of the alternative respiratory enzymes much more deeply, and in particular how the genes controlling these currently abstruse proteins are regulated in response to changing environments.

\section{Genome analysis of Aspergillus; alternative respiration pathways}

Analysis of the fully sequenced genomes of Aspergillus species (www.aspergillusgenomes.org) for alternative respiratory pathways shows the presence of homologous genes encoding multiple pathways similar to those used by $N$. crassa (Duarte et al. 2003). BlastP searching of the genomes predicts the presence of at least two AoxA (Pfam PF01786; Finn et al. 2010) enzymes in each fully sequenced Aspergillus genome (9 species: A. nidulans, A niger, A. terreus, A.

oryzae, A. flavus, A. clavatus, A. fumigatus) providing alternative oxidase activity. Alignment of the conserved AoxA domain exhibits high levels of amino acid identity. Analysis of the genomes for alternative NADH dehydrogenases encoded by $n d e A B$ and ndiA also revealed high levels of 
conservation and genome synteny across the Aspergilli (Fig. 2). The ndeAB NADH dehydrogenases are contained within a large family of class I and class II oxidoreductases (Pfam PF07992), which exhibit a characteristic NADH binding domain within a larger FAD binding motif. The alternative NADH dehydrogenases in Aspergillus encoded by ndiA are also highly conserved throughout the genus with high levels of genome synteny and high levels of amino acid identity (Pfam PF01512). The conservation of these alternative respiration pathways both at the sequence and genome level indicate the fundamental role these enzymes play in the growth of Aspergillus in the natural environment in response to oxidative stress and provide useful roles in growth during industrial bioprocesses.

Insert Figure 2 here

\section{Conclusion}

The fungi are at the heart of modern biotechnology. Although most fungal bioprocesses are highly aerobic, our understanding of fungal stress responses to high levels of $\mathrm{O}_{2}$ is far from complete. One major area needing research is the role of the alternative respiratory pathway in such conditions. It is apparent that the enzymes of this pathway (AOX and NADH dehyrogenases) are profoundly affected by oxidative stress. This in turn may lead to minimisation of ROS effects, but at the expense of major metabolic changes in fungal cultures, including decreased ATP supply and enhanced heat evolution. Our knowledge of how fungi regulate the relative flux through each pathway is minimal. Aside from the pressing need to understand more about this neglected area of fungal metabolism in our fermenters, this is a 
potentially rewarding target for metabolic engineering of fungal strains optimised for the fermenter environment.

\section{References}

Abbott DA, Suir E, Duong GH, de Hulster E, Pronk JT, van Maris AJA (2009) Catalase overexpression reduces lactic acid-induced oxidative stress in Saccharomyces cerevisiae. Appl Environ Microbiol 75:2320-2325

Angelova MB, Pashova SB, Spasova BK, Vassilev SV, Slokoska LS (2005) Oxidative stress response of filamentous fungi induced by hydrogen peroxide and paraquat. Mycol Res 109:150158

Avril M, Hathaway MJ, Cartwright MM, Gose SO, Narum DL, Smith JD (2009) Optimizing expression of the pregnancy malaria vaccine candidate, VAR2CSA in Pichia pastoris. Malar J 8:143

Bai Z, Harvey LM, McNeil B (2003a) Oxidative stress in submerged cultures of fungi Crit Rev Biotechnol 23:267-302

Bai Z, Harvey LM, McNeil B (2003b) Physiological responses of chemostat cultures of Aspergillus niger (B1-D) to simulated and actual oxidative stress. Biotechnol Bioeng 82:691-701

Bai Z, Harvey LM, White S, McNeil B (2004) Effects of oxidative stress on production of heterologous and native protein, and culture morphology in batch and chemostat cultures of Aspergillus niger B1-D. Enzym Microb Technol 34:10-21 
Baurain D et al. (2003) Regulation of the alternative oxidase Aox1 gene in Chlamydomonas reinhardtii. Role of the nitrogen source on the expression of a reporter gene under the control of the Aox1 promoter. Plant Physiol 13:1418-1430

Branduardi P, Fossati T, Sauer M, Pagani R, Mattanovich D, Porro D (2007) Biosynthesis of vitamin C by yeast leads to increased stress resistance. PLoS One 2: e1092

Carneiro P, Duarte M, Videira, A (2004) The main external alternative NAD(P)H dehydrogenase of Neurospora crassa mitochondria. Biochim Biophys Acta 1608:45-52

Cherry, J (2003) Directed evolution of industrial enzymes: an update. Curr Opin Biotechnol $14: 438-443$

Duarte M, Peters M, Schulte U, Videira A (2003) The internal alternative NADH dehydrogenase of Neurospora crassa mitochondria. Biochem J 371:1005-1011

Durocher Y and Butler M (2009) Expression systems for therapeutic glycoprotein production, Curr Opin Biotechnol 20:700-707

Fang J, Beattie DS (2003) External alternative NADH dehydrogenase of Saccharomyces cerevisiae: a potential source of superoxide. Free Rad Biol Med 34:478-488

Gasser B, Mattanovich D (2007) Antibody production with yeasts and filamentous fungi: on the road to large scale? Biotechnol Letter 29:201-212 
Guerrero-Castillo S, Vázquez-Acevedo M, González-Halphen D, Uribe-Carvajal S

In Yarrowia lipolytica mitochondria, the alternative NADH dehydrogenase interacts specifically with the cytochrome complexes of the classic respiratory pathway. Biochim Biophys Acta $1787: 75-85$

Harding HP, Zhang Y, Zeng H, et al. (2003) An integrated stress response regulates amino acid metabolism and resistance to oxidative stress. Mol Cell 11:619-633

Haynes CM, Titus EA, Cooper AA, (2004) Degradation of misfolded proteins prevents ERderived oxidative stress and cell death. Mol Cell 15:767-776

Hattori T, Kino K, Kirimura K (2009) Regulation of alternative oxidase at the transcription stage in Aspergillus niger under the conditions of citric acid production. Curr Microbiol 58:321-325

Jaszek M, Grzywnowicz K, Malarczyk E, Leonowicz A (2006) Enhanced extracellular laccase activity as a part of the response system of white rot fungi: Trametes versicolor and Abortiporus biennis to paraquat-caused oxidative stress conditions. Pesticide Biochem Physiol 85:147-154

Jones MG (2007) The first filamentous fungal genome sequences: Aspergillus leads the way for essential everyday resources or dusty museum specimens? Microbiology 153:1-6

Joseph-Horne T, Babij J, Wood, PM, Hollomon, D, Sessions RB (2000) New sequence data enable modelling of the fungal alternative oxidase and explain an absence of regulation by pyruvate. FEBS Lett 481:141-146 
Joseph-Horne T, Hollomon DW, Wood PM (2001) Fungal respiration: a fusion of standard and alternative components. Biochim Biophys Acta 1504:179-195

Juarez O, Guerra G, Martinez F , Pardo JP (2004) The mitochondrial respiratory chain of Ustilago maydis. Biochim Biophys Acta 1658:244-251

Juarez O, Guerra G, Velazquez I, Flores-Herrera O, Rivera-Perez RE and Pardo JP (2006) The physiologic role of alternative oxidase in Ustilago maydis. FEBS J 273:4603-4615

Karaffa L, Vaczy K, Sandor E, Biro S, Szentirmai A, Pocsi I (2001) Cyanide-resistant alternative respiration is strictly correlated to intracellular peroxide levels in Acremonium chrysogenum. Free Radical Res 34:405-416

Kerscher SJ (2000) Diversity and origin of alternative NADH:ubiquinone oxidoreductases. Biochim Biophys Acta 1459:274-283

Kerscher SJ, Okun JG., Brandt U (1999) A single external enzyme confers alternative NADH:ubiquinone oxidoreductase activity in Yarrowia lipolytica. J Cell Sci 112:2347-2354

Kreiner M, Harvey LM, McNeil B (2003) Morphological and enzymatic responses of a recombinant Aspergillus niger to oxidative stressors in chemostat cultures. J Biotechnol 10:251260

Li Q, Abrashev R, Harvey LM, McNeil B (2008a) Oxidative stress-associated impairment of glucose and ammonia metabolism in the filamentous fungus, Aspergillus niger B1-D. Mycol Res 112:1049-55 
Li Q, Harvey LM, McNeil B (2008b) Oxygen enrichment effects on protein oxidation, proteolytic activity and the energy status of submerged batch cultures of Aspergillus niger B1-D. Proc Biochem 43:238-246

Li Q, Harvey LM, McNeil B (2009) Oxidative stress in industrial fungi. Crit Rev Biotechnol 29 $: 199-213$

Li Y, Trush MA (1998) Diphenyleneiodonium, an NAD(P)H oxidase inhibitor, also potently inhibits mitochondrial reactive oxygen species production. Biochem Biophys Res Commun 253: 295-299

Magnani T, Soriani FM, Martins VP, Nascimento AM, Tudella VG, Curti C, Uyemura SA (2007) Cloning and functional expression of the mitochondrial alternative oxidase of Aspergillus fumigatus and its induction by oxidative stress. FEMS Microbiol Lett 271:230-238

Medentsev AG, Arinbasarova AY, Akimenko VK (2004) Reactivation of the alternative oxidase of Yarrowia lipolytica by nucleoside monophosphates. FEMS Yeast Res 5:231-236

Melo AMP, Duarte M, Videira A (1999) Primary structure and characterisation of a 64 kDa $\mathrm{NADH}$ dehydrogenase from the inner mitochondrial membrane of Neurospora crassa mitochondria. Biochim Biophys Acta 1412:282-287

Minagawa N, Koga S, Nakano M, Sakajo S, Yoshimoto A (1992) Possible involvement of superoxide anion in the induction of cyanide-resistant respiration in Hansenula anomala. FEBS Lett 302:217-219 
Mogi T, Murase Y, Mori M, Shiomi K, Omura S, Paranagama MP, Kita K (2009) Polymyxin B identified as an inhibitor of alternative $\mathrm{NADH}$ dehydrogenase and malate: quinone oxidoreductase from the Gram-positive bacterium Mycobacterium smegmatis. J Biochem 146: $491-499$

Moore AL, Albury MS, Crichton PG, Affourtit C (2002) Function of the alternative oxidase: is it still a scavenger? Trends Plant Sci 7:478-81

Murakami K , Yoshino M (1997) Inactivation of aconitase in yeast exposed to oxidative stress. Biochem Mol Biol Int 41:481-486

O'Donnell A (2007) Physiological responses to oxidative environments in submerged cultures of a filamentous fungus A. niger B1-D. PhD Thesis; University of Strathclyde, Glasgow, UK

O'Donnell A, Bai Z, McNeil B, Harvey LM (2007) Introduction to bioreactors of shake-flask inocula leads to development of oxidative stress in Aspergillus niger. Biotechnol Lett 29:895-900

Pel HJ, de Winde JH, Archer DB, et al. (2007) Genome sequencing and analysis of the versatile cell factory Aspergillus niger CBS 513.88. Nat Biotechnol 25:221-231

Perez-Torrado R, Gomez-Pastor R, Larsson C, Matallana E (2009) Fermentative capacity of dry active wine yeast requires a specific oxidative stress response during industrial biomass growth. Appl Microbiol Biotechnol 81:951-960 
Prömper C, Schneider R, Weiss H (1993) The role of the proton-pumping and alternative respiratory chain NADH:ubiquinone oxidoreductases in overflow catabolism of Aspergillus niger. Eur J Biochem 216:223-230

Raimondi S, Zanni E, Talora C, Rossi M, Palleschi C, Uccelletti D (2008) SOD1, a new Kluyveromyces lactis helper gene for heterologous protein secretion. Appl Environ Microbiol $74: 7130-7137$

Roberts TH, Rasmusson AG , Møller IM (1996) Platanetin and 7-iodo- acridone-4-carboxylic acid are not specific inhibitors of respiratory $\mathrm{NAD}(\mathrm{P}) \mathrm{H}$ dehydrogenases in potato tuber mitochondria. Physiol Plant 96:263-267

Sakajo S, Minagawa N, Yoshimoto A (1997) Effects of nucleotides on cyanide-resistant respiratory activity in mitochondria isolated from antimycin A-treated yeast Hansenula anomala. Biosci Biotechnol Biochem 61:396-399

Schmidt FR (2004) Recombinant expression systems in the pharmaceutical industry. Appl Microbiol Biotechnol. 65:363-372

Schoonooghe S, Kaigorodov V, Zawisza M, Dumolyn C, Haustraete J, Grooten J, Mertens N (2009) Efficient production of human bivalent and trivalent anti-MUC1 Fab-scFv antibodies in Pichia pastoris. BMC Biotechnol 9: Article number 70 
Seo BB, Marella M, Yagi T, Matsuno-Yagi A (2006) The single subunit NADH dehydrogenase reduces generation of reactive oxygen species from complex I. FEBS Lett 580:6105-6108

Siedow JN, Umbach AL (1995) Plant mitochondrial electron transfer and molecular biology. The Plant Cell 7:821-831

Sigler K, Chaloupka J, Brozmanova J, Stadler N, Höfer M (1999) Oxidative stress in microorganisms - I: microbial vs. higher cells - damage and defences in relation to cell aging and death. Folia Microbiol 44:587-624

Sierra-Campos E, Valdez-Solana MA, Matuz-Mares D, Velazquez I, Pardo JP (2009) Induction of morphological changes in Ustilago maydis cells by octyl gallate. Microbiology 155:604-611

Sierra-Campos E, Velazquez I, Matuz-Mares D, Villavicencio-Queijeiro A, Pardo JP (2009) Functional properties of the Ustilago maydis alternative oxidase under oxidative stress conditions. Mitochondrion (Kidlington) 9:96-102

Tanton LL, Nargang CE, Kessler KE, Li QH, Nargang FE (2003) Alternative oxidase expression in Neurospora crassa. Fungal Gen Biol 39:176-190

Turrens JF, Alexandre A, Lehninger AL (1985) Ubisemiquinone is the electron donor for superoxide formation by complex III of heart mitochondria. Arch Biochem Biophys 237:408-414 
Umbach AL, Siedow JN (2000) The cyanide-resistant alternative oxidases from the fungi Pichia stipitis and Neurospora crassa are monomeric and lack regulatory features of the plant enzyme. Arch Biochem Biophys 378:234-245

Uribe D, Khachatourians GG (2008) Identification and characterization of an alternative oxidase in the entomopathogenic fungus Metarhizium anisopliae. Can J Microbiol 54:119-127

Ukibe K, Hashida K, Yoshida N, Takagi H (2009) Metabolic engineering of Saccharomyces cerevisiae for astaxanthin production and oxidative stress tolerance. Appl Environ Microbiol 75: $7205-7211$

Veiga A, Arrabaca JD, Loureiro-Dias MC (2003) Stress situations induce cyanide-resistant respiration in spoilage yeasts. J Appl Microbiol 95:364-371

Videira A, Duarte M (2002) On complex I and other NADH:ubiquinone reductases of Neurospora crassa mitochondria. J Bioenerg Biomemb 33:197-203

Wongwicharn A, McNeil B, Harvey LM (1999) Effect of oxygen enrichment on morphology, growth, and heterologous protein production in chemostat cultures of Aspergillus niger B1-D. Biotechnol Bioeng 65:416-424

Yamawaki S, Matsumoto T, Ohnishi Y, Kumada Y, Shiomi N, Katsuda T, Lee EK, Katoh S (2007) Production of single-chain variable fragment antibody $(\mathrm{scFv})$ in fed-batch and continuous culture of Pichia pastoris by two different methanol feeding methods. J Biosci Bioeng 104:403-407 
Yukioka H, Inagaki S, Tanaka R, Katoh K, Miki N, Mizutani A, Masuko M (1998) Transcriptional activation of the alternative oxidase gene of the fungus Magnaporthe grisea by a respiratory-inhibiting fungicide and hydrogen peroxide. Biochim Biophys Acta 1442:161-169

Yurimoto H, Sakai Y (2009) Methanol-inducible gene expression and heterologous protein production in the methylotrophic yeast Candida boidinii. Biotechnol Appl Biochem 53:85-92 
Fig 1 The respiratory chain for filamentous fungi. The arrows indicate the flow of electrons. UQ, ubiquinone/ubiquinol; NDE1, external alternative $\mathrm{NAD}(\mathrm{P}) \mathrm{H}$ dehydrogenase 1; NDE2, external alternative $\mathrm{NAD}(\mathrm{P}) \mathrm{H}$ dehydrogenase 2; NDI1, internal alternative NADH dehydrogenase 1; AOX, alternative oxidase; Cyt $c$, Cytochrome $c$; I, II, III, IV, complex I, II, III, IV. (Joseph-Horne et al. 2000; Duarte et al. 2003; Carneiro et al. 2004).

Fig. 2 Genome Analysis of alternative respiratory pathways.

A: Schematic representation of syntenous regions surrounding aoxA (AN2099: A. nidulans, A niger, A. flavus, A. clavatus, A. fumigatus), ndeA (AN7500; A. nidulans, A niger, A. terreus, A. oryzae, A. flavus, A. clavatus, A. fumigatus) and ndiA (AN5629; A. nidulans, A niger, A. terreus, A. oryzae, A. flavus, A. clavatus, A. fumigatus) based on annotation at WwW.aspergillusgenome.org.

B: A ClustalW alignment (http://www.ebi.ac.uk/Tools/clustalw2/index.html) of AoxA homologues from various Aspergillus genomes compared with that of Penicillium chrysogenum indicating the high levels of homology in the PF01786 domain across the filamentous fungi (Uniprot accession numbers follow the species names due to the presence of two homologues in each genome).

C: A ClustalW alignment of NdeA homologues from four Aspergillus genomes indicating high levels of homology in the PF07992 domain.

D: A ClustalW alignment of NdiA homologues from four Aspergillus genomes indicating high levels of homology in the PF01512 domain. 


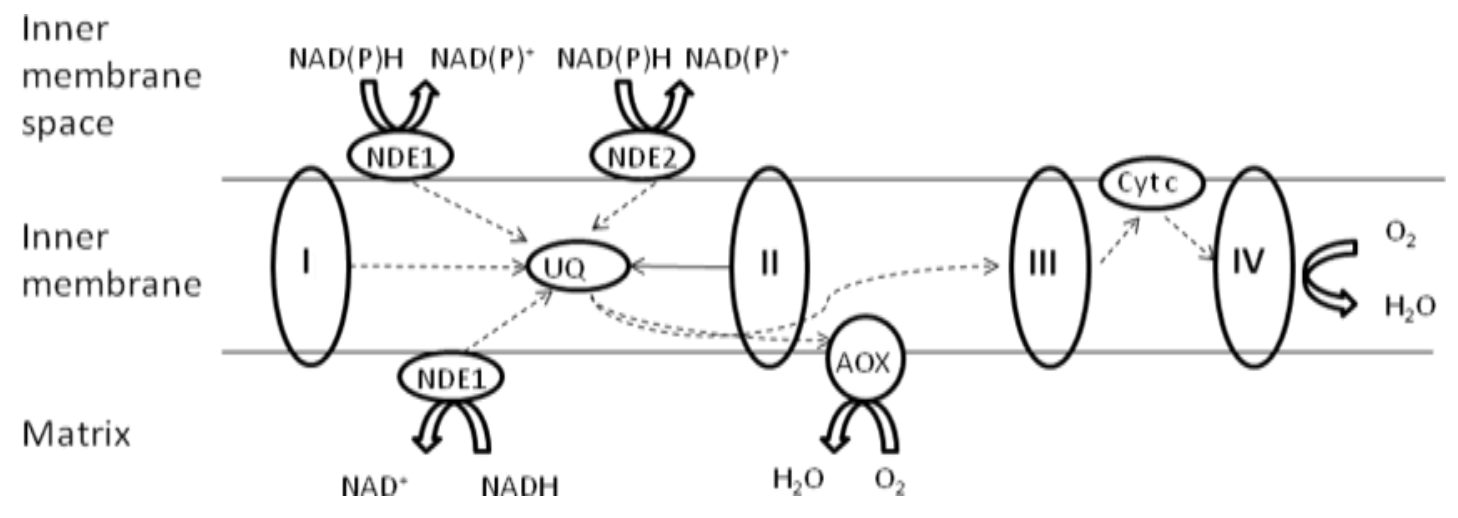


HP IOOXA HP HP aOXAgene order

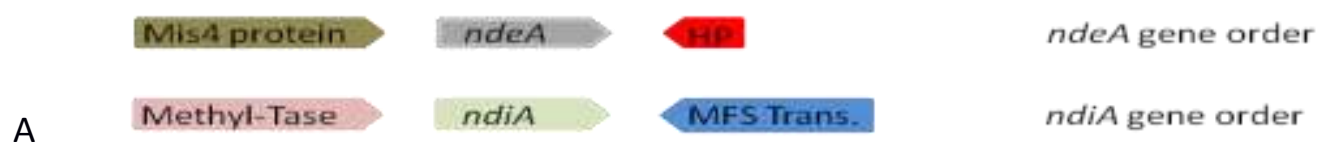

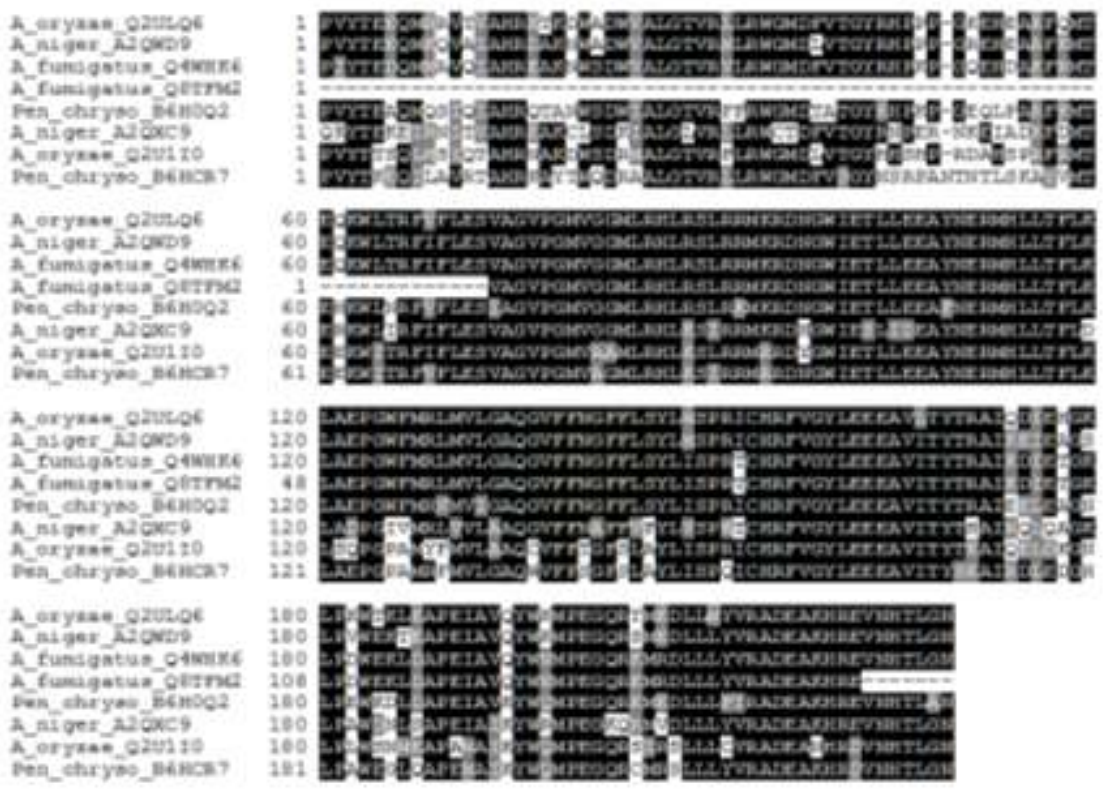

B 

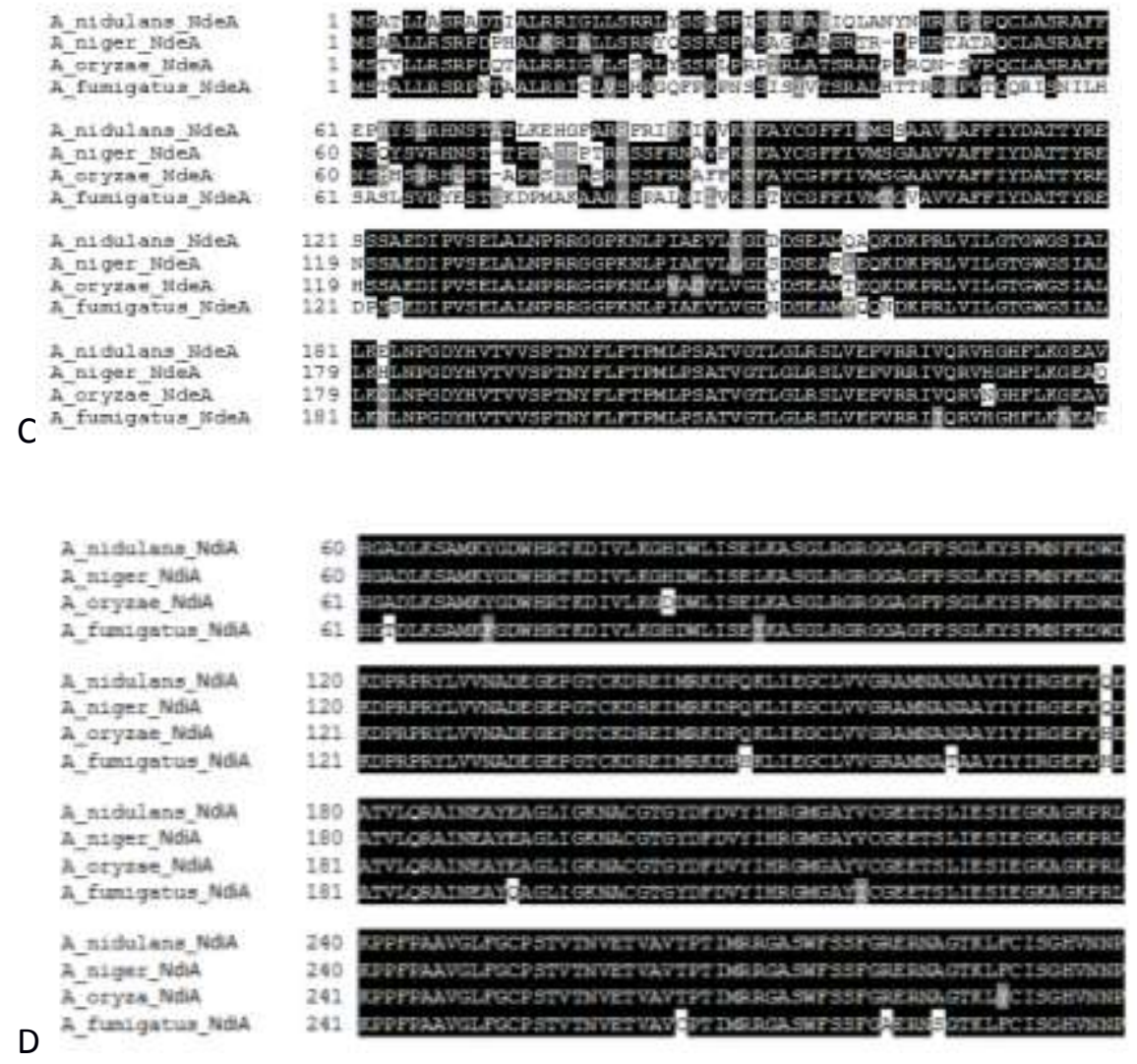